\title{
Frequency of Electrolyte Disorders and Its Effect On Mortality Among Children Admitted In Pediatric Intensive Care Unit
}

\author{
Maryam Haider, Ammara Hameed, Sara Fatima, Meher Afroze, Nadeem Noor, Uzma Arshad
}

\section{ABSTRACT}

Objective: To determine the frequency of electrolyte disorders, i.e., serum sodium and potassium and to evaluate its effect on mortality rate among children admitted at the pediatric intensive care unit.

Study Design and Setting: This was a descriptive cross sectional study conducted at Pediatric Intensive care unit of Civil Hospital Karachi from April to December 2017

Methodology: Informed consent was obtained from 150 parents of the children who fulfill the inclusion criteria. Laboratory data (serum sodium and serum potassium) were recorded during the stay in the pediatric intensive care unit. Data was analyzed using SPSS version 20. Mean \pm S.D was calculated for quantitative variables. Frequency and percentage were calculated for gender, electrolyte disorders and mortality. Effect modifiers were controlled by stratification of age, gender and electrolyte disorders (Hypernatremia, Hyponatremia, Hyperkalemia, and Hypokalemia). Post-stratification, Chi-squared test was applied. P-value $=0.05$ was taken as significant.

Results: Out of 150 patients, electrolyte disorders in terms of serum sodium and potassium, were found in $86(57.3 \%)$ children. Mortality in children with electrolyte disorders was found to be $46(53.5 \%)$ which was significantly higher (P<0.001) than patients without electrolyte disorders 40(46.5\%). Hypernatremia was found in 48(32\%), hyponatremia $24(16 \%)$, hyperkalemia 21(14\%) and hypokalemia in 42(28\%) patients.In comparison; of electrolyte disorders with mortality; significant association was found in hypernatremia $(\mathrm{P}<0.001)$, and hyperkalemia $(\mathrm{P}<0.001)$.

Conclusion: The most common electrolyte abnormalities were hypernatremia and hypokalemia. Mortality was significantly higher in subjects with electrolyte disorders, especially hypernatremia and hyperkalemia.

Keywords: Electrolyte Disorders, Intensive care unit (ICU), Mortality, Potassium, Sodium

\section{INTRODUCTION:}

Electrolytes such as sodium and potassium play a vital role in maintaining cellular structure and function, generating energy and controlling the potential for action across cell membranes, especially those of muscles and nerve cells, which play an important role in critically ill patient's outcome. ${ }^{1,2}$ Any disruption in the serum level of these

I Maryam Haider
Assistant Professor, Department of Paediatrics
Jinnah Medical College Hospital, Karachi
I Email: dr.maryamhaider@ hotmail.com
Ammara Hameed
Senior Registrar, Department of Paediatrics
Bahria University Medical \& Dental College
Sara Fatima
Paediatrics Emergency Medicine Fellow,
Agha Khan University Hospital,
I Marachi
I Assistant Professor, Department of Paediatrics,
I Jinnah Medical College Hospital, Karachi
I Nadeem Noor
Issistant Professor, Department of Paediatrics,
Jinnah Medical College Hospital, Karachi
I Uzma Arshad
I Assistant Professor, Department of Paediatrics,
Innah Medical College Hospital, Karachi
I Received: 06-Feb-2020
I Accepted: 22-Apr-2020

electrolytes may lead to a variety of clinical disorders including neuromuscular dysfunction and life-threatening cardiac arrhythmias, particularly where more than one electrolyte is deficient. ${ }^{3,4}$ The most severe complications of hypokalemia are cardiac arrhythmia and acute respiratory failures due to respiratory muscles paralysis while other adverse effects include muscle weakness, fatigue, rhabdomyolysis, nausea and constipation. ${ }^{5}$ Although hyperkalemia is associated with neuromuscular symptoms such as extreme muscle weakness or ascending muscle paralysis, it can also cause paresthesiaas, palpitation, and cardiac conduction that can lead to adverse outcome in patients in intensive care unit patients. ${ }^{7}$ Hyponatremia is also a frequent electrolyte abnormality observed in critically ill patients. ${ }^{8,9}$ Severe acute decline in serum sodium concentration may cause neurologic dysfunction such as seizures and coma. The most severe complication of hyponatremia is cerebral edema that may lead to catastrophic conditions like brain stem herniation and cardiac arrest ${ }^{10}$. On the other hand; hypernatremia can cause brain hemorrhage like subdural and subarachnoid hemorrhages due to the rupture of bridging veins because of a decrease in brain volume. The clinical symptoms are progressive that might commence with irritability, agitation, lethargy, and irritability and can progress to twitching, seizures, and coma ${ }^{11}$. Thus, maintaining the levels of potassium and sodium within acceptable limits is therefore well understood in most 
intensive care units. They are known as prognostic predictors and their disruption can be avoided by considering the appropriate intravenous fluids and nutrition ${ }^{12}$.

Ample literature on electrolyte imbalance have concentrated on the occurrence and prevalence of such electrolyte disorders in patients in the intensive care unit but there is scarce literature on the impact of such disorders on mortality rate. Pakistan has a greater number of children with electrolyte disorders admitted every day to pediatric intensive care unit. Despite of this, the research on the prevalence of electrolyte disorders and its effect on mortality rates are lacking within our population. In fact, due to a lack of resources and clinical expertise about the treatment of electrolyte disorders, mortality in our population is estimated to be higher than in the western world. ${ }^{13,14}$ Therefore, the aim of this study was not only to assess the frequency of serum sodium and potassium disorders but also to assess their effects on mortality rate in children admitted to the pediatric intensive care unit in Civil Hospital Karachi. The outcome of this study would be beneficial to take aggressive measures against electrolyte imbalances to reduce the mortality.

\section{METHODOLOGY:}

All children between the ages of 2 months to 12 years who were admitted in Pediatric Intensive Care Unit, Civil Hospital Karachi from April to December 2017 with duration of at least 48 hours of PICU stay, were included in the study. Patients undergoing emergency or elective surgical procedures, patients on diuretic therapy and patients with documented chronic or co-morbid illness were excluded such as endocrinopathy, cerebral palsy, chronic liver disease, congenital heart disease. Patients were enrolled through non-probability consecutive sampling. The demographic data of all the participants regarding age, sex and admitting diagnosis was noted on a predesigned proforma. Blood samples were drawn on admission to find out the initial serum levels of sodium, potassium. Then, the levels were repeated 24 hourly or earlier (if needed). Determination of electrolytes was the standard examination in all patients admitted to the pediatric intensive care unit at Civil Hospital, Karachi. No personal identity revelation was made on the questionnaires and confidentiality of all data was maintained during the study phases. There is no conflict of interest or funding source to declare. The normal serum levels of sodium and potassium are given below ${ }^{15}$.

Normal serum sodium level 135mEq/L-145 mEq/L.

Normal serum potassium level $3.4 \mathrm{mEq} / \mathrm{L}-5.3 \mathrm{mEq} / \mathrm{L}$.

Any value below or above the following cut-off values was considered to be anomalous suggesting an electrolyte imbalance.

For this study, Mortality was recorded for those patients, who died after at least 48 hours of admission in Pediatric Intensive care unit. Sample size of the study $(n=142)$ was calculated by taking prevalence of hypernatremia i.e. $6.3 \% .^{16}$ Confidence Interval of $95 \%$ and margin of error $4 \%$. For convenience, a total of 150 patients were enrolled. Data was analyzed using SPSS version 20 . Mean \pm S.D was calculated for quantitative variables. Frequency and percentage were calculated for gender, diagnosis at admission, electrolyte disorders and mortality. Effect modifiers were controlled by stratification of age, gender and electrolyte disorders (Hypernatremia, Hyponatremia, Hyperkalemia, and Hypokalemia). Post-stratification, Chi-squared test was applied. P-value $=0.05$ was taken as significant.

\section{RESULTS:}

A total number of 150 patients were included in this analysis with a mean age of 50.33 months \pm 46.46 (Table.1) with Confidence Interval (42.84-57.83). Out of these 150 patients, $93(62 \%)$ were male and 57 (38\%) were female.Regarding the admitting diagnosis, majority i.e. $52(34.66 \%)$ had respiratory illness, 36(24\%) had neurological illness, 28 (18.66\%) had sepsis/infection, 12 (8\%) had cardiovascular problem, $10(6.66 \%)$ had gastrointestinal ailment and 12(8\%) were categorized in miscellaneous group including endocrine diseases.

Mixed electrolyte disorders (serum sodium and potassium abnormality) were found in $86(57.33 \%$ ) children. Hypernatremia was found in $48(32 \%)$, Hyponatremia 24(16\%), Hyperkalemia 21(14\%) and Hypokalemia was found in 42(28\%) children. Hypernatremia and Hyperkalemia were significantly more common in patients who expired $(62.5 \%)(\mathrm{P}=0.0001)$, and $(28.8 \%)(\mathrm{P}=0.0001)$ respectively (Figure2 \& 3).

Overall, mortality was found to be $34.66 \%$ (52 out of 150 patients). Mortality in children with electrolyte disorders was found to be $46(53.5 \%$ ) which was significantly higher $(\mathrm{P}=0.0001)$ than children without electrolyte disorders 40(46.5\%)(Figure1).

\section{DISCUSSION:}

This comprehensive case study was carried out at Civil Hospital Karachi to assess the prevalence of electrolyte disorders and their impact on mortality in children admitted in pediatric intensive care unit.

Fluids and electrolyte disturbances are one of the most common clinical problems encountered in the intensive care unit. Disturbances in fluid and electrolyte homeostasis can occur in critical conditions such as severe burns, sepsis, trauma, heart failure and brain damage. In most cases, electrolyte disturbances may remain subtle and may lead to higher mortality and morbidity. ${ }^{17}$

In our study, mixed electrolyte disorders were found in $86(57.33 \%)$ children. Since the four electrolyte disorders (Hypo/Hypernatremia, Hypo/Hyperkalemia) were considered together and imbalance among any of them was noted, this might be the reason for the high incidence. Cummings BM 
Maryam Haider, Ammara Hameed, Sara Fatima, Meher Afroze, Nadeem Noor, Uzma Arshad

Table 1. Demographic characteristics and main clinical variables $(n=150)$

\begin{tabular}{|l|c|c|}
\hline Parameter & Frequency & Percentage\% \\
\hline Mean Age(in months) & $55.33 \pm 46.46$ & \\
\hline Gender & & \\
\hline Boy & 93 & 62 \\
\hline Girls & 57 & 38 \\
\hline Admitting diagnosis & & \\
\hline Respiratory & 52 & 34.66 \\
\hline CNS & 36 & 24 \\
\hline Sepsis/infection & 28 & 18.66 \\
\hline CVS & 12 & 8 \\
\hline GIT & 10 & 6.66 \\
\hline Miscellaneous & 12 & 8 \\
\hline Electrolyte Disorders & & \\
\hline Yes & 86 & 57.33 \\
\hline No & 64 & 42.77 \\
\hline Hypernatremia & & \\
\hline Yes & 48 & 32 \\
\hline No & 102 & 68 \\
\hline Hyponatremia & & \\
\hline Yes & 24 & 16 \\
\hline No & 126 & 84 \\
\hline Hyperkalemia & & \\
\hline Yes & 21 & 14 \\
\hline No & & 86 \\
\hline Hypokalemia & 429 & 72 \\
\hline Yes & & \\
\hline No & & \\
\hline Patients Survived & & \\
\hline Yes & & \\
\hline No & & \\
\hline
\end{tabular}

Figure 1. Comparison Of Overall Mortality With Mixed Electrolyte Disorders $(\mathrm{N}=150)$

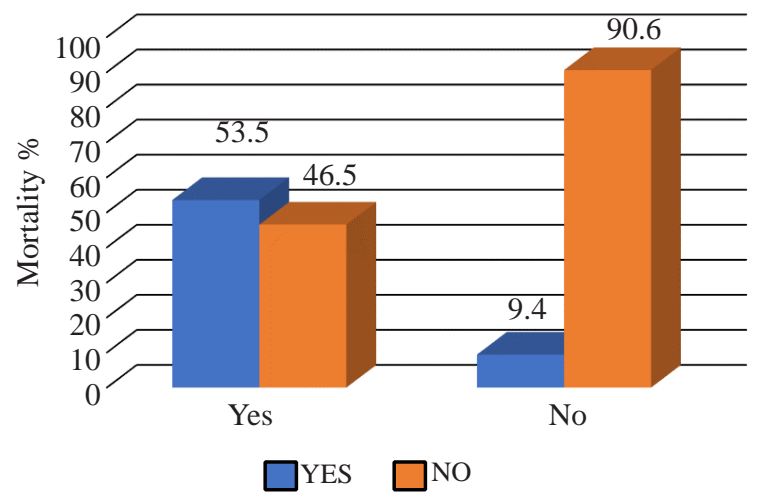

Figure 2. Comparison of mortality with serum sodium levels $(n=150)$

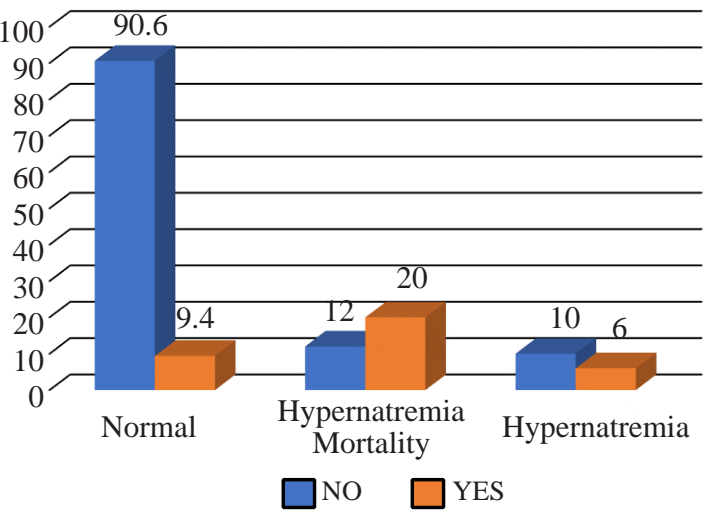

Figure 3. Comparison of mortality with serum potassium levels $(n=150)$

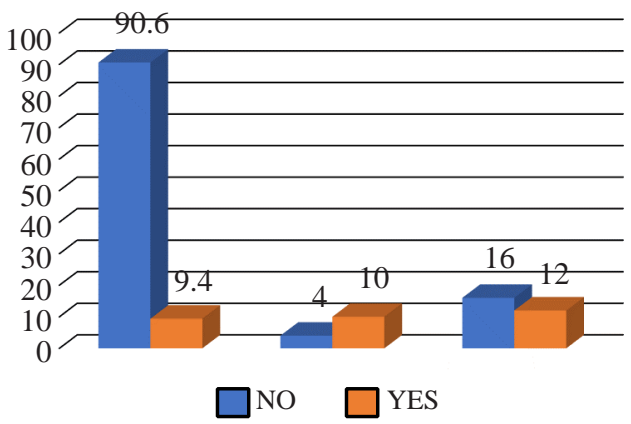

found potassium abnormalities alone to be around $40 \% .^{18}$ Another study focused at all five electrolytes showed $60 \%$ incidence. ${ }^{2}$ This proves the statement that electrolyte abnormalities are very common in critically ill children.

Majority had respiratory (34.66\%), neurological (24\%) and infective/sepsis $(18.66 \%)$ etiology as noted in previous studies. ${ }^{2,190}$ The reason for less number of gastrointestinal cases $(3.96 \%)$ could be the initial stabilization in ER and later shifting to ward upon improvement thus bypassing the PICU stay. This is important because gastroenteritis in children is the major source of electrolyte imbalance, but prompt and proper treatment may avoid PICU admission.

A study conducted in Kathmandu identified hyponatremiato be $56 \%$, followed by hypokalemia $46 \%$. Almost $37 \%$ of patients had mixed electrolyte imbalances ${ }^{21}$. However, our study showed hyponatremia $(16 \%)$, hypokalemia $(28 \%)$ and mixed electrolyte disorders $(57.33 \%)$

In another study, published in India, mixed electrolyte imbalances were observed in $33 \%$ of children. The most frequent electrolyte imbalance was Hyperkalemia which was found in $14.4 \%$ similar to our study in which hyperkalemia was found in $14 \%$ patients. Hyponatremia was found to be $6.9 \%$ compared to $16 \%$ of hyponatremia in our study ${ }^{22}$. 
Another prospective study showed that severe hyponatremia was associated with three fold increase risk of morbidity and mortality ${ }^{23}$.

Most of the literature has reported hyponatremia to be more prevalent than hypernatremia i.e. $23.2 \%$ vs. $16.7 \%{ }^{15}, 27.43 \%$ vs. $3.5 \%{ }^{6}, 50.5 \%$ vs. $9.4 \%^{2}$. Sachdev A noted hyponatremia to be $19.3 \%^{20}$. However, hypernatremia was found in $48 \%$ of cases and hyponatremia in $16 \%$ cases. This might be due to the institutional policy of using $0.9 \%$ dextrose saline as maintenance intravenous fluid rather than half strength or other hypotonic solutions in children above one month of age.

One research concluded that potassium imbalance is frequent among children admitted in PICU. Hyperkalemia was observed in $5.4 \%$ of 727 children enrolled in this study, while in our study hyperkalemia was reported in $14 \%$. This variation in prevalence could be due to the fact that in this study hyperkalemia was defined as a serum potassium value $>6 \mathrm{mEq} / \mathrm{L}$. However, in this study serum potassium levels $>5.3 \mathrm{mEq} / \mathrm{L}$ are considered to be hyperkalemia. ${ }^{24}$

In a study conducted by Kathryn Maitland, it was found that extreme hyperkalemia wasassociated with falciparum malaria in 9 children (16\%), of whom 7 (78\%) died shortly after $\operatorname{admission}^{25}$.

Overall mortality in this study was $34.66 \%(52 / 150)$. However, mortality documented by Panda I was $23.73 \%{ }^{6}$ and by Jan $\mathrm{M}$ et al as $22.8 \%{ }^{\mathbf{2 6}}$.

In one local study, results showed that out of 101 children enrolled, electrolyte imbalances were present in 85 (84.15\%) children. Among these patients, the most frequent abnormality noted was hypocalcemia which was seen in 49 (57.64\%) patients, followed by hypernatremia in 32 (37.64\%) cases and hypophosphatemia in $30(35.29 \%)$ patients. Hypokalemia was observed in $26(30.58 \%)$ and hypermagnesemia in 18 $(21.17 \%)$ children. The study found that electrolyte imbalances in critically ill children are associated with a higher risk of mortality. Early mortality, i.e. within 48 hours of admission, was reported in 14 cases of which 12 had electrolyte disturbances. ${ }^{27}$.

In another study carried out at a hospital in India in PICU, results revealed that out of 132 children enrolled in the study, $71(53.79 \%)$ had an electrolyte abnormalities. Among these patients, $29(40.85 \%)$ had hyponatremia, 9 (12.68\%) had hypernatremia, $13(18.31 \%)$ had hyperkalemia, $8(11.27 \%)$ had hypokalemia. They also found that patients with electrolyte abnormalities had relatively longer hospital stays. $^{28}$ The limitation of this study was the smaller sample size; a study with large sample size with multiple centers in Pakistan to validate the results of should be conducted in future. Electrolyte disturbances in children become obvious during illness and early detection and management of these derangements is essential to avoid serious life-threatening situation. They are considered as significant and reliable indicator of mortality as shown by this study. Another limitation as this study was that the mortality might be primarily due to underlying clinical disorder and not the electrolyte disturbances. For this purpose, children with documented chronic or co-morbid illnesses should have been excluded and mortality was recorded only in those patients with duration of PICU stay of at least 48 hours. Serial serum electrolytes tests are helpful in preventing severe life-threatening circumstances. Thus, the need for early interventions to correct these imbalances is imperative in reducing the mortality.

\section{CONCLUSION:}

The results of this study showed higher mortality in children with electrolyte disorders especially hypernatremia and hyperkalemia, thus, concluding that electrolyte imbalance was a significant predictor of prognosis in seriously ill patients.

\section{REFERENCES:}

1. Sedlacek M, Schoolwerth AC, Remillard BD. Electrolyte disturbances in the intensive care unit. Semin Dial 2006;19(6):496-501.

2. Agarwal N, Rao Y, Saxena R, Acharya R. Profile of serum electrolytes in critically ill children: A prospective study. Indian J child Health. 2018;5(2):128-132.

3. Rukesh CC, Shalini B. Correlation between serum electrolytes and clinical outcome in children admitted to PICU. IOSR J Dent Med Sci. 2017;16(11):24-27.

4. Ducceschi V, D’Andrea A, Liccardo B, Sarubbi B, Ferrara L, Romano GP, Santangelo L, Iacono A, Cotrufo M. Ventricular tachyarrhythmias following coronary surgery: predisposing factors. International journal of cardiology. 2000 Mar 31;73(1):43-8.

5. Lee JW. Fluid and electrolyte disturbances in critically ill patients. Electrolytes \& Blood Pressure. 2010 Dec 1;8(2):7281.

6. Panda I, Save S. Study of association of mortality with electrolyte abnormalities in children admitted in pediatric intensive care unit. Int J ContempPediatr. 2018;5:1097-1103.

7. Viera AJ, Wouk N. Potassium disorders: hypokalemia and hyperkalemia. American family physician. 2015 Sep 15;92(6):487-95.

8. Friedman B, Cirulli J. Hyponatremia in critical care patients: frequency, outcome, characteristics, and treatment with the vasopressin V2-receptor antagonist tolvaptan. Journal of critical care. 2013 Apr 1;28(2):219-e1.

9. Amin A, Deitelzweig S, Christian R, Friend K, Lin J, Belk $\mathrm{K}$, Baumer D, Lowe TJ. Evaluation of incremental healthcare resource burden and readmission rates associated with hospitalized hyponatremic patients in the US. Journal of hospital medicine. 2012 Oct;7(8):634-9.

10. Kilic O, Demirkol D, Ucsel R, Citak A, Karabocuoglu M. Hypophosphatemia and its clinical implications in critically ill children: a retrospective study. Journal of critical care. 2012 Oct 1;27(5):474-9.

11. Adrogue HJ, Madias NE. Hypernatremia. New England Journal of Medicine. 2000 May 18;342(20):1493-9. 
12. Reddy A, Thapar RK, Gupta RK. Electrolyte disturbances in critically ill children admitted to pediatric tertiary care centre. J Evol Med Dent Sci. 2017;6:3269-3273.

13. Syed M, Khawaja FB, Saleem T, Khalid U, Rashid A, Humayun KN. Clinical profile and outcomes of paediatric patients with diabetic ketoacidosis at a tertiary care hospital in Pakistan. Journal of the Pakistan Medical Association. 2011;61(11):1082.

14. Wasay M, Zaidi S, Jooma R. Non communicable diseases in Pakistan: burden, challenges and way forward for health care authorities.

15. Elala G, Shimelis D. Patterns of electrolyte abnormalities in children $0-15$ years of age admitted to pediatric emergency and intensive care units of a tertiary hospital. IOSR Journal of Dental and Medical Sciences. 2018;17(2):12-6.

16. Mousavi SA, Shahabi S, Mostafapour E, Purfakharan M, Fereshtehnejad SM, Amini J, Khojandi M, Raji H. Comparison of the serum electrolyte levels among patients died and survived in the intensive care unit. Tanaffos. 2012;11(4):36.

17. Marshall DC, Salciccioli JD, Goodson RJ, Pimentel MA, Sun KY, Celi LA, Shalhoub J. The association between sodium fluctuations and mortality in surgical patients requiring intensive care. Journal of critical care. 2017 Aug 1;40:63-8.

18. Cummings BM, Macklin EA, Yager PH, Sharma A, Noviski N. Potassium abnormalities in a pediatric intensive care unit: frequency and severity. J Intensive Care Med. 2014;29(5):269-274.

19. Haque A, Bano S. Improving outcome in pediatric intensive care unit in academic hospital in Pakistan. Pak J Med Sci. 2009;25(4):605-608.
20. Sachdev A, Pandharikar N, Gupta D, Gupta N, Gupta S, Venkatraman ST. Hospital acquired hyponatremia in pediatric intensive care unit. Indian J Crit Care Med. 2017; 21:599-603.

21. Shah GS, Das BK, Kumar S, Singh MK, Bhandari GP. Acid base and electrolyte disturbance in diarrhoea. Kathmandu University medical journal (KUMJ). 2006 Dec;5(17):60-2.

22. Subba Rao SD, Thomas B. Electrolyte abnormalities in children admitted to pediatric intensive care unit. Indian pediatrics. 2000;37(12):1348-53.

23. Singhi S, Prasad SV, Chugh KS. Hyponatremia in sick children: a marker of serious illness. Age. 1994 Jan;125:126-30.

24. Singhi S, Gulati S, Prasad SV. Frequency and significance of potassium disturbances in sick children. Indian pediatrics. 1994 Apr;31(4):460.

25. Maitland K, Pamba A, Fegan G, Njuguna P, Nadel S, Newton CR, Lowe B. Perturbations in electrolyte levels in Kenyan children with severe malaria complicated by acidosis. Clinical infectious diseases. 2005 Jan 1;40(1):9-16.

26. Jain M, Sha A, Parajapati R. Study of electrolyte imbalance in critically ill children. Int J Int Med Res. 2015;2(2):56-59.

27. Naseem F, Saleem A, Mahar IA, Arif F. Electrolyte imbalance in critically ill paediatric patients. Pakistan journal of medical sciences. $2019 \mathrm{Jul} ; 35(4): 1093$.

28. Chary DC, Shalini B. Correlation between serum electrolytes and clinical outcome in children admitted to PICU. IOSR Journal of Dental and Medical Sciences16. 2017; 11:24-7. 